\title{
Measurement of retinal blood flow with fluorescein leucocyte angiography using a scanning laser ophthalmoscope in rabbits
}

Yunsik Yang, Sungkang Moon, Soowhan Lee, Jaeduck Kim

\begin{abstract}
Aims-To measure blood flow in the rabbit retinal circulation with fluorescein leucocyte angiography using a scanning laser ophthalmoscope.

Methods-Blood was withdrawn from the ear vein of a rabbit (New Zealand White), mixed with fluorescent dye in a test tube and centrifuged. The yellow-brown layer containing fluorescein stained leucocytes was collected and injected into the ear vein of the same rabbit while performing fluorescein angiography with a scanning laser ophthalmoscope. The image of retinal angiography displaying circulating fluorescent leucocytes was recorded on video tape. From each frame of the video tape, the consecutive positions of fluorescein stained leucocytes were digitised using an image analysis system and the velocity of blood flow was calculated.

Results-Fluorescent leucocytes were clearly visualised in the retinal arteries, capillaries, and veins which allowed measurement of blood flow. The mean capillary velocity was 0.69 (SD 0.21 ) $\mathrm{mm} / \mathrm{s}$. The mean velocities of leucocytes measured in different sized vessels were as follows: $5.83(2.42) \mathrm{mm} / \mathrm{s}$ in arteries over $50 \mu \mathrm{m}, 3.33(0.62) \mathrm{mm} / \mathrm{s}$ in those $35-50 \mu \mathrm{m}$, and $2.42(1.08) \mathrm{mm} / \mathrm{s}$ in arteries under $35 \mu \mathrm{m}, 3.08(1.56) \mathrm{mm} / \mathrm{s}$ in veins over $50 \mu \mathrm{m}, 2.79(1.49) \mathrm{mm} / \mathrm{s}$ in those 35-50 $\mu \mathrm{m}$, and $1.21(0.50) \mathrm{mm} / \mathrm{s}$ in veins under $35 \mu \mathrm{m}$. Blood flow pulsation occurs in arteries, arterioles, veins, and venules but not capillaries.

Conclusion-Fluorescein leucocyte angiography can be used for simultaneous measurement of the blood flow in retinal arteries, veins, and capillaries.

(Br F Ophthalmol 1996; 80: 475-479)
\end{abstract}

Department of Ophthalmology School of Medicine Wonkwang University, lksan City, Chonbuk, Korea

Y Yang

Y Yaon

S Moon
S Lee

J Kim

Correspondence to: Yunsik Yang, MD, Department of

Ophthalmology, School of Ophthalmology, Schoo Medicine, Wonkwang dong, Iksan City, Chonbuk, dong, Iksan City,

Accepted for publication 21 December 1995

Two recent developments of the scanning laser ophthalmoscope (SLO 101, Rodenstock, Germany) enabled us to observe fluorescent dots in retinal vessels, especially in juxtafoveal capillaries. ${ }^{12}$ The scanning laser ophthalmoscope is equipped with laser light and a confocal system. ${ }^{3}$

Many methods have been used to measure retinal blood flow. In the past, the blue field entoptic technique ${ }^{4}$ was used for measuring the perifoveal capillary leucocyte velocity but it has become unpopular owing to the subjective nature of the examination. More recently, the fluorescent dye dilution technique ${ }^{5}$ and quantitative fluorescein angiography ${ }^{6-8}$ have been utilised to assess retinal blood flow but they tend to produce unclear images resulting in accurate measurement. Using the scanning laser ophthalmoscope, the velocity of blood flow can be monitored in the perifoveal capillary ${ }^{129}$ immediately after the injection of fluorescein dye. The targeted dye delivery method $^{1011}$ was also devised to measure retinal blood flow but it is not popular owing to its technical difficulties. Laser Doppler velocimetry ${ }^{12}$ has become an effective technique to measure blood flow but only in the large retinal vessels. The fluorescent vesicle system, ${ }^{13}$ which measures retinal blood flow by injecting fluorescent dye containing liposomes into the vein, is similar to our study, but it must be calibrated carefully and orientated precisely. Recently, Nishiwaki et $a l^{14}$ and Kimura et al ${ }^{15}$ reported leucocyte haemodynamics with acridine orange staining methods in cynomolgus monkeys and rats. However, acridine orange is toxic to humans and so blood vessels cannot be visualised. In this study, we have tested the feasibility of a simple method which can be used for monitoring retinal blood flow. In this method, the conventional fluorescein angiography had been modified so that blood is stained externally and reintroduced by injection into the same animal. The fluorescein dye typically stains leucocytes and platelets among blood components. In conventional angiography using the scanning laser ophthalmoscope, fluorescent leucocytes are invisible in large vessels because the fluorescent cells are floating in the highly fluorescent plasma, although they can be visualised in the macular capillaries. We eliminated the problems of highly fluorescent plasma by re-introducing only externally stained leucocytes into the systemic circulation.

In contrast with other methods, fluorescein leucocyte angiography can be used to measure retinal blood flow more objectively for a longer time and can be easily used in humans.

Table 1 Number of leucocytes whose velocity was measured in different vessels in seven rabbits $(A-G)$

\begin{tabular}{lcccr}
\hline Rabbits & $\begin{array}{l}\text { Arteries } \\
\text { (centreline) }\end{array}$ & $\begin{array}{l}\text { Veins } \\
\text { (centreline) }\end{array}$ & Capillaries & Total \\
\hline A & $3(3)$ & $5(4)$ & 2 & 10 \\
B & $5(4)$ & $2(1)$ & 1 & 8 \\
C & $3(2)$ & $6(2)$ & 1 & 10 \\
D & $13(7)$ & $6(2)$ & 0 & 19 \\
E & $9(6)$ & $9(3)$ & 4 & 22 \\
F & $9(6)$ & $9(4)$ & 1 & 19 \\
G & $8(1)$ & $8(2)$ & 3 & 19 \\
Total & $50(29)$ & $40(18)$ & 12 & 102 \\
\hline
\end{tabular}



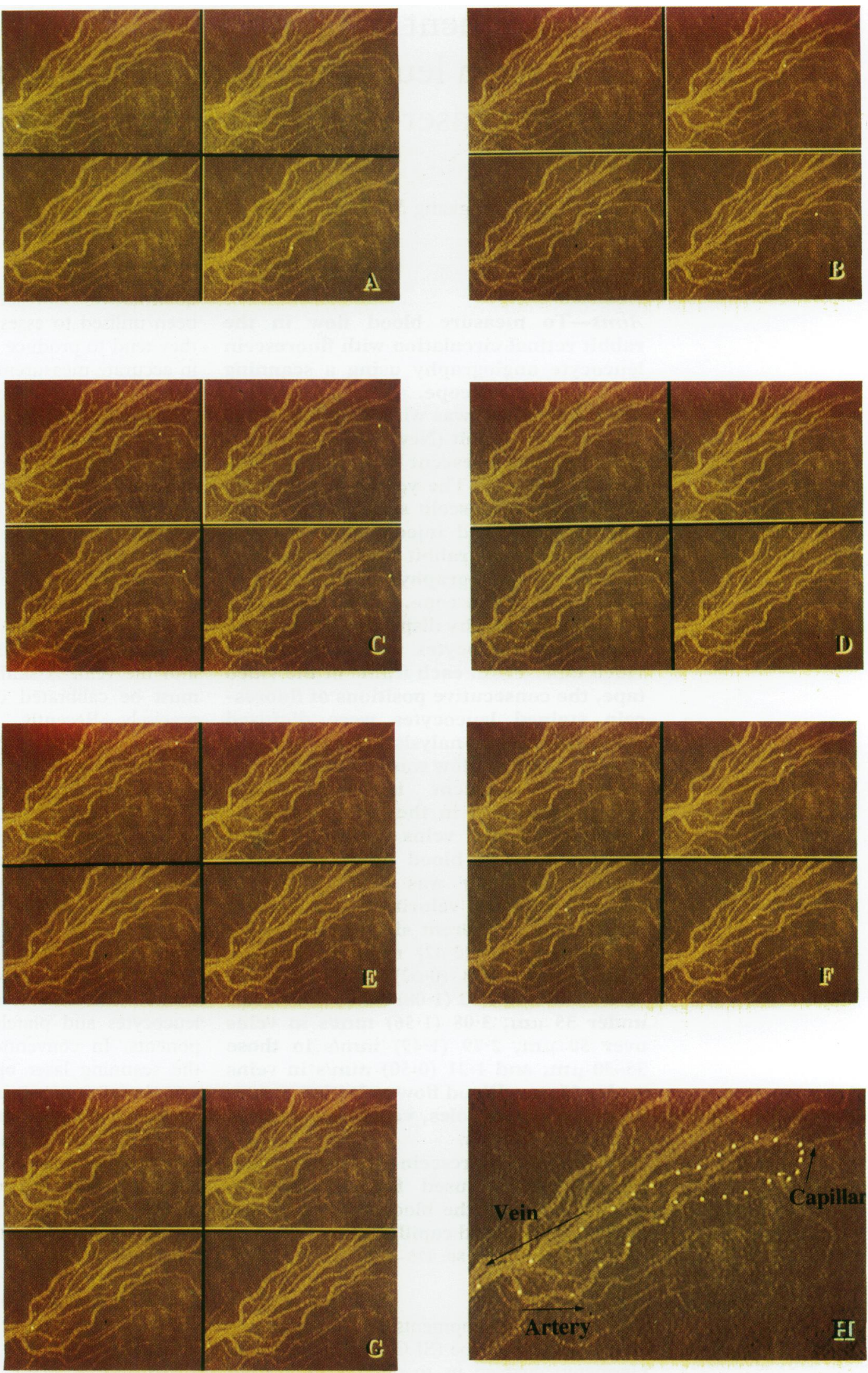

Figure 1 Trace of fluorescent dots in artery, capillary, and vein. (A-G) Consecutive image at an interval of $1 / 10$ second in artery, capillary, and vein. (H) Overlaid image of fluorescent dots from $A$ to $G$.

\section{Subjects and methods}

Seven rabbits (New Zealand White, ordered from $\mathrm{A}$ to $\mathrm{G}$ ) of about $2 \mathrm{~kg}$ body weight were used in this study. Two $\mathrm{ml}$ of blood was sampled from an ear vein and mixed with $0.3 \mathrm{ml}$ of fluorescein dye solution (10\% fluorescein sodium) in a sterile test tube. It was then centrifuged at $3000 \mathrm{rpm}$ for 5 minutes and the
9/20 fraction of fluorescent plasma and erythrocyte bulk were removed. The remnant was the yellow-brown layer of leucocyte fluid with a trace of plasma and erythrocytes. After mydriasis with $0.5 \%$ tropicamide ophthalmic solution, the leucocyte fluid was injected into an ear vein. Then, fluorescein angiography was performed using the scanning laser ophthalmoscope. 


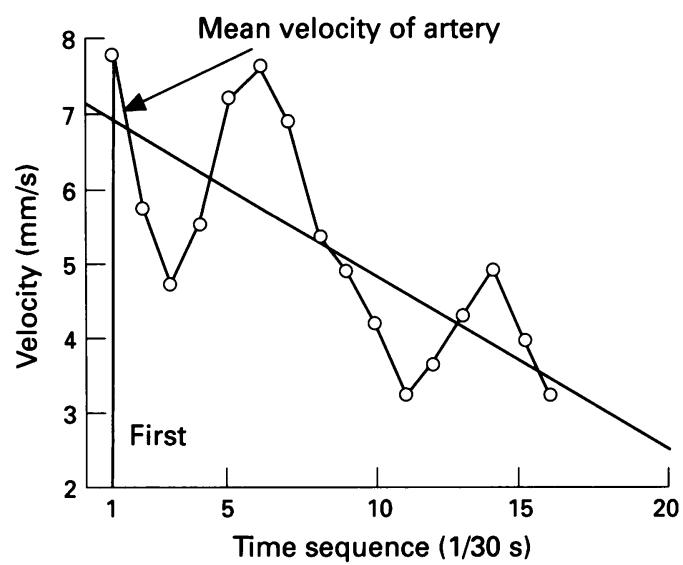

Figure 2 Velocity of leucocytes in two cycles of pulsation in artery is plotted. Mean velocity is obtained with crossing point of linear regression curve and first time sequence.

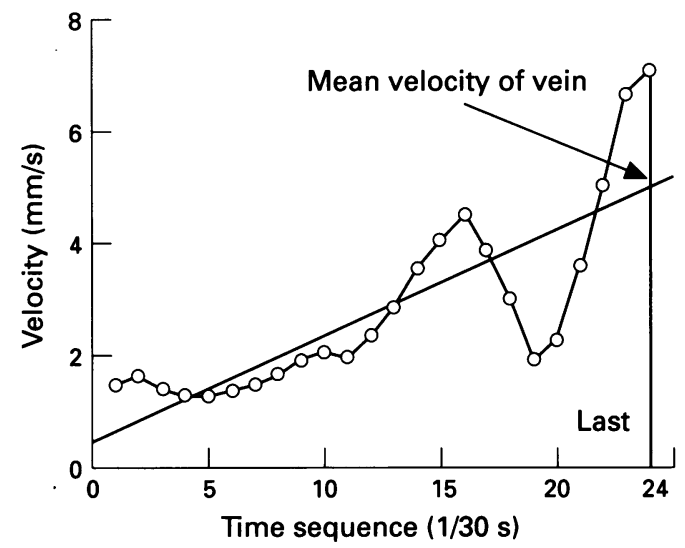

Figure 3 Velocity of leucocytes in three cycles of pulsation in vein is plotted. Mean velocity is obtained with crossing point of linear regression curve and last time sequence.

We analysed the image with an image processing system in the form of a National Television Standards Committee-type video signal, which was recorded on a super-VHS video tape. Fluorescent dots flowing from arteries via capillaries to veins were observed clearly. Angiographic images were obtained by recording 30 frames of picture per second $(60 \mathrm{~Hz})$. Picture frames were digitised using a frame grabber $\mathrm{A}-\mathrm{V}$ board included in the Power Macintosh 7100/66AV (software Video Monitor, simple QuickTime Apple

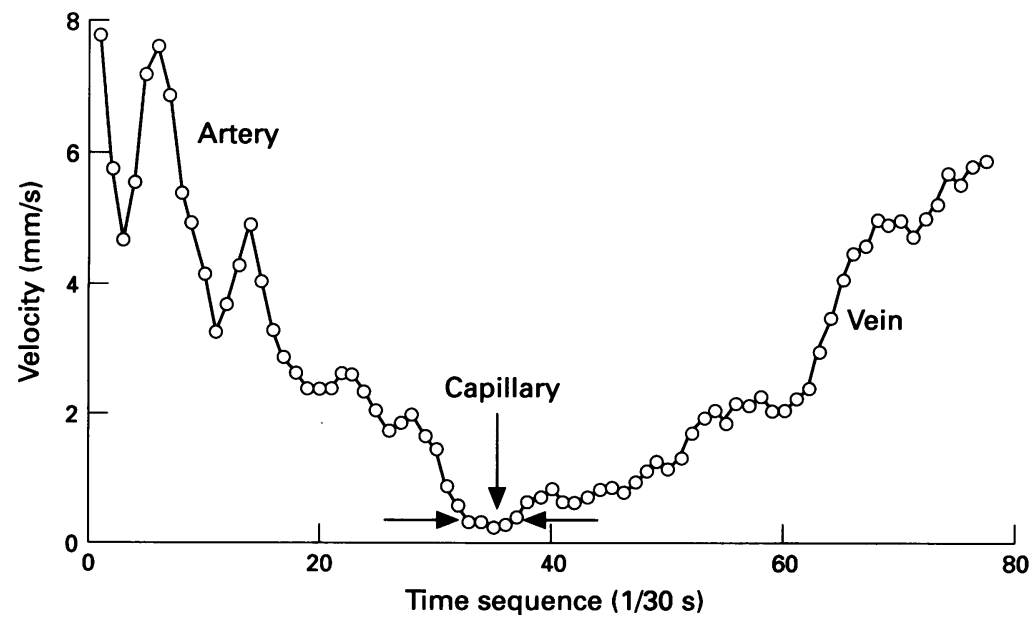

Figure 4 Effects of pulsation of retinal blood flow in artery and vein are plotted against time. Capillary identification is based on this plotted graph. Capillaries have the lowest velocity and have no pulsation.

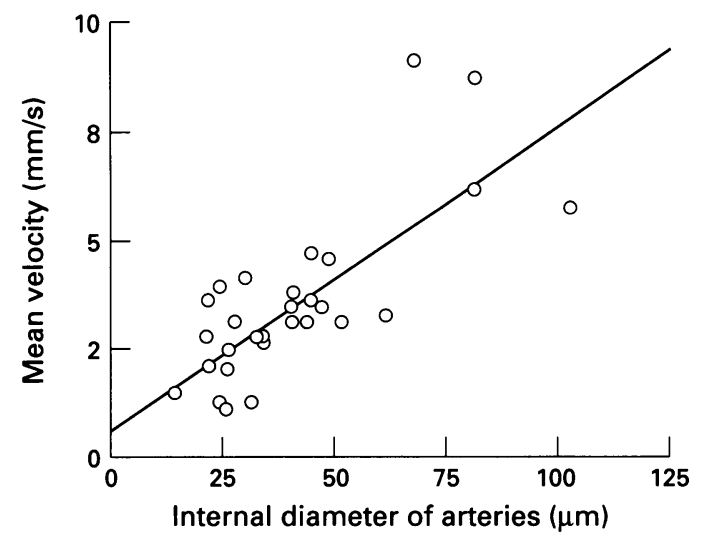

Figure 5 Relation between velocity of blood flow in centre of vessels and the internal diameter of the arteries.

Computer Inc, 1993). The distances travelled by a fluorescent dot on consecutive pictures were measured using an image analysis program (software IMAGE by Wayne Rasband, NIH Research Service Branch, version-1.37).

Real retinal size was calculated according to the Littman method. ${ }^{16}$ It was confirmed in vivo after insertion of a thin wire ruler on to the surface of the retina using scanning laser ophthalmoscope (this was done because the real retinal size of the rabbit is different from that of the human). The mean real retinal size of $1^{\circ}$ was $0.163 \mathrm{~mm}$. The number of pixels in the $16^{\circ} \times 12^{\circ}$ size of image was $640 \times 480$. The real size of one pixel was $4 \cdot 1 \mu \mathrm{m}$ in a $20^{\circ}$ angle. In the $40^{\circ}$ angle one pixel was $8.2 \mu \mathrm{m}$. The number of leucocytes whose velocity was measured was 50 (centreline, 29) in the arteries, 40 (centreline, 18) in the veins, and 12 in the capillary (Table 1). The diameters of retinal vessels varied in all animals. The speeds of blood flow also varied greatly depending on centrality of laminar flow. Therefore, it was impossible to compare the data across the seven animals. Data were analysed according to the size of vessel and centrality of laminar flow in each vessel. The time interval between each frame was $1 / 30$ second. Consecutive images were superimposed so that the movement of the same fluorescent dot could be traced on one image (Fig 1). Distances between dots marked on the superimposed images were then measured. The mean velocity was calculated with the linear regression curve of one to three cardiac cycle pulsations in

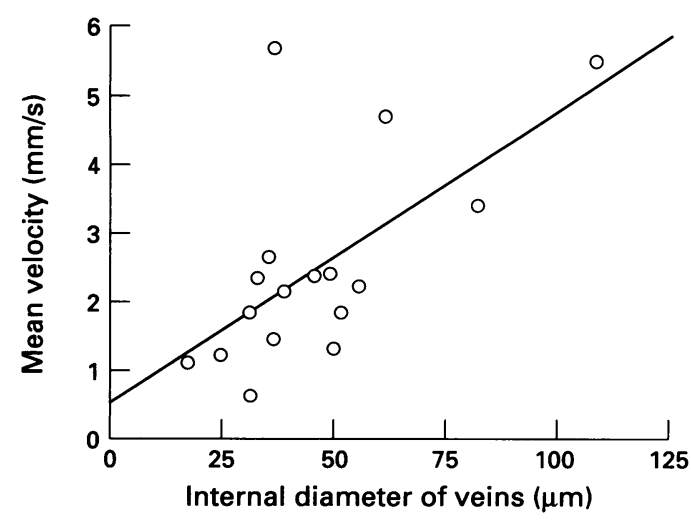

Figure 6 Relation between velocity of blood flow in centre of vessels and the internal diameter of the veins. 
Table 2 Mean (SD) centreline leucocyte velocity according to vessel thickness

\begin{tabular}{lllll}
\hline $\begin{array}{l}\text { Thickness of } \\
\text { vessels }(\mu \mathrm{m})\end{array}$ & $\begin{array}{l}\text { Arteries } \\
(\mathrm{mm} / \mathrm{s})\end{array}$ & $\begin{array}{l}\text { No of } \\
\text { measurements }\end{array}$ & $\begin{array}{l}\text { Veins } \\
(\mathrm{mm} / \mathrm{s})\end{array}$ & $\begin{array}{l}\text { No of } \\
\text { measurements }\end{array}$ \\
\hline$\geqslant 50$ & $5.83(2.42)$ & 7 & $3.08(1.56)$ & 7 \\
$35-50$ & $3.33(0.62)$ & 10 & $2.79(1.49)$ & 6 \\
$\leqslant 35$ & $2.42(1.08)$ & 12 & $1.21(0.50)$ & 4
\end{tabular}

the artery (Fig 2) and vein (Fig 3). Capillary identification was based on the plotted graph (Fig 4). Capillaries had the lowest velocity through the vessels and no pulse.

\section{Results}

Fluorescent leucocytes were clearly visualised in the retinal arteries, capillaries, and veins for at least 2 hours which enabled us to measure their blood flow. Even the pulsation of blood flow was observed in the arteries, arterioles, veins, and venules but not in the capillaries (Fig 4). The mean velocity in the capillaries was $0.69(\mathrm{SD} 0.21) \mathrm{mm} / \mathrm{s}$. The mean velocities of the blood flow measured in the centre of the vessels are shown in Figures 5 and 6. The mean velocities of leucocytes measured in different sizes of vessels were as follows: $5.83(2.42) \mathrm{mm} / \mathrm{s}$ in arteries over $50 \mu \mathrm{m}, 3.33$ $(0.62) \mathrm{mm} / \mathrm{s}$ in those $35-50 \mu \mathrm{m}$, and 2.42 $(1.08) \mathrm{mm} / \mathrm{s}$ in those under $35 \mu \mathrm{m} ; 3.08(1.56)$ $\mathrm{mm} / \mathrm{s}$ in veins over $50 \mu \mathrm{m}, 2.79(1.49) \mathrm{mm} / \mathrm{s}$ in those $35-50 \mu \mathrm{m}$, and $1 \cdot 21(0.50) \mathrm{mm} / \mathrm{s}$ in those under $35 \mu \mathrm{m}$ (Table 2).

We examined the fluorescein stained leucocyte fluid with a fluorescent microscope before it was injected into the ear vein. This clearly demonstrated highly fluorescent leucocytes and platelets, however, erythrocytes were not stained at all (Fig 7). Fluorescent leucocytes in the peripheral blood smear were pale at 12 hours after injection of fluorescein dye in conventional fluorescein angiography; however, leucocyte fluorescence with fluorescein leucocyte angiography was more fluorescent than with fluorescein angiography (Fig 8).

\section{Discussion}

Scanning laser ophthalmoscopy ${ }^{17}$ provides high resolution images - that is, time resolution of $1 / 30$ second in $60 \mathrm{~Hz}$ area and space resolution of $8 \mu \mathrm{m}$. In fluorescein angiography, the scanning laser ophthalmoscope provides us

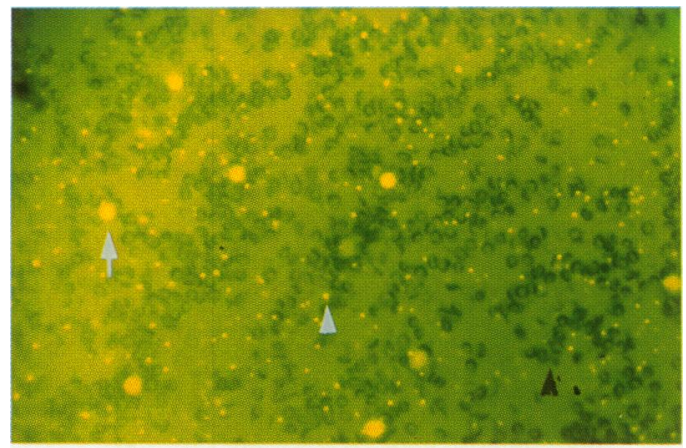

Figure 7 Microscopic picture of leucocyte fluid (400X). White arrow points to fluorescent leucocytes, black arrowhead indicates unstained erythrocyte, and white arrowhead indicates small fluorescent platelet. with high resolution images, enabling us to see a fluorescein image under low intensity illumination. This process requires a much smaller amount of fluorescein dye injection compared with the conventional fundus camera.

Fluorescein leucocyte angiography is based on the differential fluorescent staining among blood components. The fluorescent leucocytes were collected by centrifugation. Since this technique needs external manipulation of blood samples, the sampled blood was carefully manipulated under sterile conditions. Absence of cell destruction or blood coagulation was confirmed by microscopic examination. This study does not explain the mechanism of leucocyte staining with fluorescein dye and staining differences between in vitro and in vivo conditions. In this study, only prolongation of leucocyte fluorescence in fluorescein leucocyte angiography is revealed.

In previous fluorescein angiography using a scanning laser ophthalmoscope, it was not confirmed that the fluorescent dots in perifoveal capillary were leucocytes. In this study, we found that leucocytes were the source of fluorescence in perifoveal capillaries and large vessels. Tanaka et al ${ }^{9}$ reported that the fluorescent dots were leucocytes, plasma, or platelet aggregates. Fluorescent dots seen in this study cannot be platelets or platelet aggregates since these dots were uniform in size and were much larger than single platelets. The possibility that the dots seen in this study were plasma was ruled out since the amount of plasma introduced with the leucocytes into the blood stream was very small and it was diluted. Therefore, it is most likely that the fluorescent
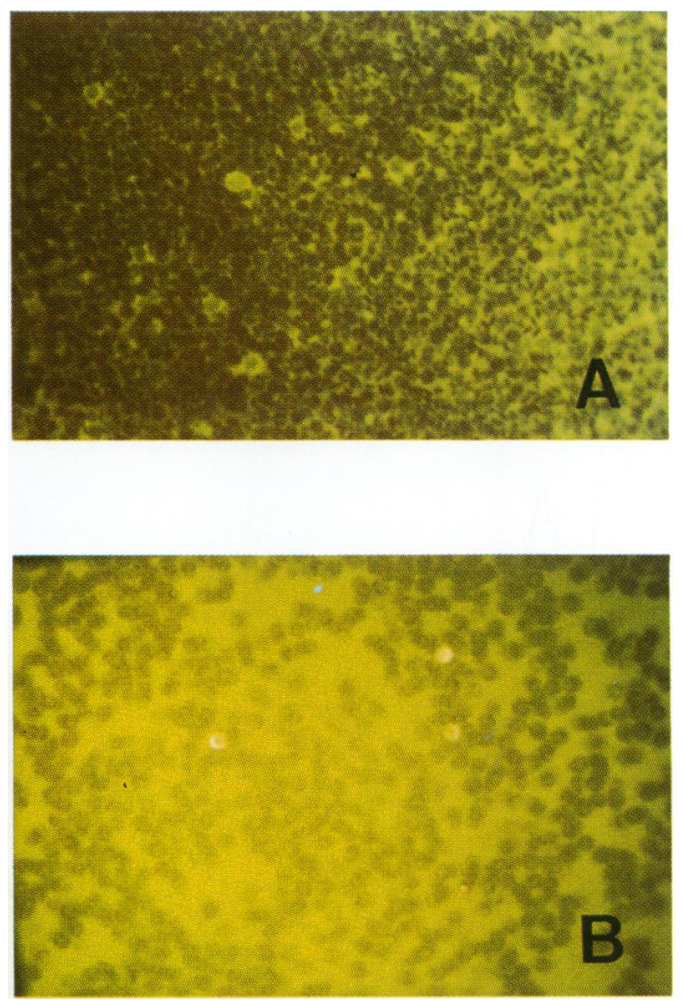

Figure 8 Microscopic picture of peripheral blood smear 12 hours after dye injection in fluorescein angiography and fluorescein leucocyte angiography. $(A)$ Leucocytes are pale in fluorescein angiography. (B) Leucocyte fluorescence is more prolonged in fluorescein leucocyte angiography. 
dots observed in the blood vessels were fluorescent stained leucocytes.

As described above, this technique is capable of recognising pulsatile flow in the retinal vessels which show a repeating pattern of longer and longer distances between dots, followed by a period of shorter and shorter distances between dots. The range is up to 250 per minute, so one cardiac cycle consisted of eight consecutive frames in this study.

In summary, fluorescent leucocyte angiography has advantages over other methods of measuring blood flow. Using this method, retinal blood flow can be measured in all areas of the retina. Also, macrocirculation and microcirculation can be measured simultaneously. Furthermore, the blood flow can be monitored at various intervals during the cardiac cycle and blood flow can be measured at any distance from the vessel wall.

In addition, this method can be used clinically because it does not require injection of liposome or fluorescent vesicles into the blood circulation. However, its limitations for clinical application are (1) difficulty in maintaining sterility during external manipulation of the blood and (2) the possibility of blood cell destruction during centrifugation. Also, large amounts of blood for sampling may be needed. If these problems can be overcome, then this method can be applied to humans.

Presented at the 5th international meeting on scanning lase ophthalmoscopy, tomography, and microscopy, San Antonio, Texas, 26-28 October 1995.

1 Wolf S, Arend O, Toonen H, Eng D, Bertram B, Jung F, et al. Retinal capillary blood flow measurement with scanning laser ophthalmoscope. Ophthalmology 1991; 98: 996-1000.
2 Ohnishi Y, Fujisawa K, Ishibashi T, Kojima H. Capillary blood flow velocity measurements in cystoid macular edema with the scanning laser ophthalmoscope. $A m \mathcal{F}$ Ophthalmol 1994; 17: 24-9.

3 Woon WH, Fitzke FW, Bird AC, Marshall J. Confocal imaging of the fundus using a scanning laser ophthalmoscope. Br F Ophthalmol 1992; 76: 470-4.

4 Fallon TJ, Chowiencyzk P, Kohner EM. Measurement of retinal blood flow in diabetes by the blue-light entoptic phenomenon. Br $f$ Ophthalmol 1986; 70: 43-6.

5 Riva CE, Feke GT, Ben-Sira I. Fluorescein dye-dilution technique and retinal circulation. Am $\mathcal{F}$ Physiol 1978; 234: H315-22.

6 Koyama T, Matsuo N, Shimizu K, Mihara M, Tsuchida Y, Wolf $\mathrm{S}$, et al. Retinal circulation timed in quantitative fluorescein angiography. Graefes Arch Clin Exp Ophthalmol 1990; 228: 442-6.

7 Jung F, Kiesewetter H, Körber N. Wolf S, Reim M, Müller G. Quantification of characteristic blood-flow parameters in the vessels of the retina with a picture analysis system for video-fluorescence angiograms: initial findings. Graefes Arch Clin Exp Ophthalmol 1983; 221: 133-6.

8 Arch Clin Exp Ophthalmol 1983; 221: 133-6. I. Quantitative measurement of retinal blood flow in I. Quantitative measurement of retinal blood flow in
human beings by application of digital image-processing methods to television fluorescein angiograms. Graefes Arch Clin Exp Ophthalmol 1983; 221: 110-2.

9 Tanaka T, Muraoka K, Shimizu K. Fluorescein fundus angiography with scanning laser ophthalmoscope. Ophthalmology 1991; 98: 1824-9.

10 Khoobehi B, Peyman GA, Vo K. Laser-triggered repetitive fluorescein angiography Ophthalmology 1992; 99: 72-9.

11 Khoobehi B, Niesman MR, Peyman GA, Oncel M. Repetitive, selective angiography of individual vessels of the retina. Retina 1989;9: 87-96.

12 Green GJ, Feke GT, Goger DG, McMeel W. Clinical application of the laser Doppler technique for retinal blood flow studies. Arch Ophthalmol 1983; 101: 971-4. 13 Khoobehi B, Peyman GA. Fluorescent vesicle system (a new technique for measuring blood flow in the retina). Ophthalmology 1994; 101: 1716-26.

14 Nishiwaki H, Ogura Y, Kimura H, Kiryu J, Honda Y. Quantitative evaluation of leukocyte dynamics in retinal microcirculation. Invest Ophthalmol Vis Sci 1995; 36: 123-30.

15 Kimura H, Kiryu J, Nishiwaki H, Ogura Y. A new fluorescent imaging procedure in vivo for evaluation of the cent imaging procedure in vivo for evaluation of the 223-8.

16 Littmann H. Zur Bestimmung der wahren Grösse eines Objektes auf dem Hintergrund des lebenden. Augenklin Monatsbl Augenheilkd 1982; 180: 286-9.

17 Nasemann JE, Müller M. Scanning laser angiography. In: Nasemann JE, Burk ROW, eds. Scanning laser ophthalmoscopy and tomography. Munich: Quintessenz, 1990: 63-80. 